\title{
Data correlation analysis for optimal sensor placement using a bond energy algorithm
}

\author{
Wei Lu, Runfa Wen ${ }^{\mathrm{a}}$, Jun Teng ${ }^{\mathrm{a}, \mathrm{b}}$, Xiaoling $\mathrm{Li}^{\mathrm{a}}$, Chao $\mathrm{Li}^{\mathrm{a}}$ \\ ${ }^{a}$ Department of Civil and Environmental Engineering, Harbin Institute of Technology Shenzhen \\ Graduate School, Xili University Town HIT Campus, Shenzhen 518055, China \\ ${ }^{\mathrm{b}}$ College of Civil Engineering, Fujian University of Technology, No.3 Xueyuan Road University Town, \\ Fuzhou 350108, China \\ Corresponding author email: tengj@hit.edu.cn, lu.wei@hit.edu.cn
}

\begin{abstract}
Optimal sensor placement is one of the crucial and fundamental factors for constructing a cost-effective structural health monitoring system and is related to the effective evaluation of the state of the structure. Structural responses are correlated to some extent, as the structural behavior is continuous. Based on the above two considerations, the question arises of how to obtain the maximum amount of information for understanding the structure using measurements from limited sensors and not be limited to direct monitoring at the placements where the limited sensors are located. Data correlation analysis for optimal sensor placement is proposed using a bond energy algorithm, in which the objectives, such as structural response evaluation covering the maximum structural responses using measurements from sensors located at the optimal placements, are taken into account. The data correlation analysis is conducted for the structural responses, and the correlation matrix is established. Furthermore, the optimal sensor placements and the correlation of the responses at element locations can be determined using the bond energy algorithm. A Schwedler single-layer spherical lattice dome-like structure, which is a common large space steel structure, is used to simulate the structural responses and verify the effectiveness of the proposed method by discussion of different scenarios of parameter selection.
\end{abstract}

Keywords: structural health monitoring, optimal sensor placement, data correlation, bond energy algorithm

\section{INTRODUCTION}

With the development of structural health monitoring technology, more attention has been given to its application in large-span space structures that have numerous nodes and components. Considering the limitations of economic and site conditions, it is impossible to place sensors at every position [1-3]. Therefore, it has become an important research question to optimize the finite number of sensors to obtain as much information as possible regarding the structure in the field of structural health monitoring. Furthermore, such prophase work is indispensable to implementing a structural health monitoring system [4].

At present, there are many ways to obtain optimal sensor placements. Kammer [5] proposed an effective independence method of optimal sensor placements for large space structures. In this method, the position was sorted based on the contribution of linear independence of the target mode components; by deleting points with little contribution to the independence of the target modes, the limited sensors can be chosen to collect as much information as possible for obtaining modal information. The modal strain energy method was proposed to analyze the structure, in which the larger modal strain energy in terms of degrees of freedom coincides with the location of the larger structural response, and the sensors placed in these positions therefore benefit structural modal identification [6]. The spline function interpolation method is another solution to obtain the optimal sensor placements, in which the structural responses of the other points and limited measure node modal responses are first obtained. Then, the minimum interpolation error is used to determine the optimal sensor placements for a simple beam [7]. To obtain the modal dynamic information used in fingerprint update identification and model update for 
the structural health monitoring of bridges, Cui et al. [8] proposed a methodology of optimal sensor placements with the purpose of selecting key measuring points from a complex degree of freedom structural model. Guyan [9] proposed the model reduction method to determine optimal sensor placements for reflecting the low-order vibration modes of the structure. Master-slave constraint equations for degrees of freedom were substituted into the kinetic energy or strain energy expression of the system to reduce the degrees of freedom. The structural degrees of freedom can be divided into primary and secondary degrees of freedom, and the shrinkage stiffness or mass matrix can be formed while maintaining the primary degrees of freedom. Lu et al. [10] proposed a method for optimal sensor placement using a distance measure matrix and synthesized support degree, in which the number and placement of accelerometers were determined by the values of the synthesized support degree. The aforementioned optimal sensor placement methods are mostly used for modal identification, the optimal sensor placement methods were also additional studied with the aim to the structural response reconstruction and monitoring [11], such like the optimal placement of sensors for sub-surface fatigue crack monitoring [12], the sensor positioning and choice of the number of sensors were optimized in terms of the reconstruction on the temperature field considering the error propagation in case of uncertain measurements [13], the optimal sensor placement for enhancing sensitivity to change in stiffness was proposed to find the optimal configuration of sensors that would best predict structural damage [14], the optimal sensor placement methodology was proposed so as to better estimate the vibration response of the entire structure [15] and so on. The stress distribution and displacement development of the structure are important monitoring parameters for structural safety estimation and should be considered in the optimal sensor placement method for such strain or displacement sensors. For such consideration, the measurements of strain or displacement sensors can not only display the structural responses at the placement where the sensors are located but also reflect the structural responses at placements correlated with sensor locations. An additional objective of optimal sensor placement for strain or displacement sensors is to avoid redundancy among the measurements from different measuring points. Correlation is a parameter that can represent the redundancy among the measurements; higher (lower) correlations can lead to greater (less) redundancy, i.e., more (less) overlapping information was measured. Therefore, selecting measuring points with little association with each other can make it possible to gain more independent measuring information that is more comprehensive and reliable.

To reduce the redundancy information of measurement sensor systems and maximize the function of limited sensors, as well as to evaluate the structural responses with the measurements from limited sensors, this paper presents a method of optimal sensor placement based on correlation. First, a correlation matrix of potential sensor placements is established and processed into a binary matrix. Second, the potential sensor placements are preliminarily optimized and classified using the bond energy algorithm (BEA), in which the potential sensor placements in the same category are strongly correlated and the potential sensor placements in different categories are weakly correlated. Then, the final position and number of measuring points can be determined according to the proposed principle of optimal sensor placement. The optimal sensor placement in the deformation monitoring of a single-layer Schwedler reticulated dome-like steel structure is conducted to verify the effectiveness of the proposed method by analyzing the information entropy of the optimized results.

\section{DATA CORRELATION ANALYSIS OF STRUCTURAL RESPONSES}

\section{A. The establishment of the correlation matrix}

The correlation degree is usually expressed as $\gamma$, which describes the association of structure response information between two positions and reflects their correlation level. It is a non-dimensional 
parameter that ranges from -1.0 to 1.0 (including -1.0 and 1.0). The closer the absolute correlation value is to 1 , the more strongly the structural responses from two positions are correlated, whereas the closer the absolute correlation value is to 0 , the more weakly the structural responses from the two positions are correlated.

It is assumed that $X \in R^{m \times n}$ represents the response information matrix of $n$ positions in $m$ load cases, i.e.,

$$
X=\left[\begin{array}{llll}
x_{1} & x_{2} & \cdots & x_{n}
\end{array}\right]=\left[\begin{array}{cccc}
x_{11} & x_{12} & \cdots & x_{1 n} \\
x_{21} & x_{22} & \cdots & x_{2 n} \\
\vdots & \vdots & \vdots & \vdots \\
x_{m 1} & x_{m 2} & \cdots & x_{m n}
\end{array}\right]
$$

For two arbitrary positions at $i$ and $j$, the response information is $x_{i}$ and $x_{j}$, and the sample variance and covariance computation formulas are as follows:

$$
\begin{gathered}
\operatorname{Var}\left(x_{i}\right)=\frac{1}{m-1} \sum_{k=1}^{m}\left(x_{k i}-\overline{x_{i}}\right)^{2}=\sigma_{i i} \\
\operatorname{Var}\left(x_{j}\right)=\frac{1}{m-1} \sum_{k=1}^{m}\left(x_{k j}-\overline{x_{j}}\right)^{2}=\sigma_{j j} \\
\operatorname{Cov}\left(x_{i}, x_{j}\right)=\frac{1}{m-1} \sum_{k=1}^{m}\left(x_{k i}-\overline{x_{i}}\right)\left(x_{k j}-\overline{x_{j}}\right)=\sigma_{i j}
\end{gathered}
$$

where $\bar{x}_{i}=\frac{1}{n} \sum_{k=1}^{n} x_{k i}$ and $\overline{x_{j}}=\frac{1}{n} \sum_{k=1}^{n} x_{k j}$ are the mean values of $x_{i}$ and $x_{j}$, respectively, and the correlation degree formula of structural response information at positions $i$ and $j$ is:

$$
\gamma_{i j}=\operatorname{Corr}\left(x_{i}, x_{j}\right)=\frac{\operatorname{Cov}\left(x_{i}, x_{j}\right)}{\sqrt{\operatorname{Var}\left(x_{i}\right) * \operatorname{Var}\left(x_{j}\right)}}=\frac{\sigma_{i j}}{\sqrt{\sigma_{i i} * \sigma_{j j}}}
$$

The correlation matrix $R$ can be expressed as

$$
R=\left[\gamma_{i j}\right]_{n \times n}=\left[\begin{array}{cccc}
\gamma_{11} & \gamma_{12} & \cdots & \gamma_{1 n} \\
\gamma_{21} & \gamma_{22} & \cdots & \gamma_{2 n} \\
\vdots & \vdots & \vdots & \vdots \\
\gamma_{n 1} & \gamma_{n 2} & \cdots & \gamma_{n n}
\end{array}\right]
$$

\section{B. Binary processing to correlation matrix}

The correlation threshold is expressed as $\eta$; when the absolute value of the correlation is no smaller than the correlation threshold, i.e., $\left|r_{i j}\right| \geq \eta$, the element in the $\mathrm{i}^{\text {th }}$ row and $\mathrm{j}^{\text {th }}$ column of the correlation matrix is replaced by 1 , and when the absolute value of correlation is smaller than $\eta$, i.e., $\left|r_{i j}\right|<\eta$, the element in the $i^{\text {th }}$ row and $j^{\text {th }}$ column of the correlation matrix is replaced by 0 . With this binary processing of the correlation matrix, the equivalent correlation matrix $D$, whose elements are all one or zero, can be expressed as

$$
D=\left[d_{i j}\right]_{n \times n}=\left[\begin{array}{cccc}
d_{11} & d_{12} & \cdots & d_{1 n} \\
d_{21} & d_{22} & \cdots & d_{2 n} \\
\vdots & \vdots & \vdots & \vdots \\
d_{n 1} & d_{n 2} & \cdots & d_{n n}
\end{array}\right]
$$




\section{BOND ENERGY ALGORITHM FOR EQUIVALENT CORRELATION MATRIX}

\section{A. Matrix transformation using the bond energy algorithm}

The bond energy algorithm (BEA) [16] is a type of clustering approach, which can transform the matrix into submatrices with characteristics of block division by calculating the bond energy of elements into rows and columns. The BEA is used to calculate the bond energy of the rows and columns in the equivalent correlation matrix. The row and column transformation is taken subsequently based on the value of the bond energy to enable an equivalent correlation matrix with the characteristics of block division. The equivalent correlation matrix can be represented with several diagonal submatrices, whereas the positions reflecting the same diagonal submatrix are considered as one group and are more strongly correlated than the other positions. The advantage of BEA method is such equivalent correlation matrix, which can show the correlation points with the diagonal submatrices explicitly and the optimal sensor placement can be determined directly. The first step to transform the equivalent correlation matrix is to calculate the bond energy of elements in rows and columns, such as

$$
\begin{gathered}
E_{r h(h+i)}=\sum_{k=1}^{n} d_{h, k} \cdot d_{h+i, k} \\
E_{c k(k+i)}=\sum_{h=1}^{n} d_{h, k} \cdot d_{h, k+i}
\end{gathered}
$$

$E_{r h(h+i)}$ is the bond energy of row $h$ and $h+i$, where $1 \leq i \leq n-h$, and $E_{c k(k+i)}$ is the bond energy of column $k$ and $k+i$, where $1 \leq i \leq n-k$. The flow chart of the bond energy transformation is shown in figure 1 .

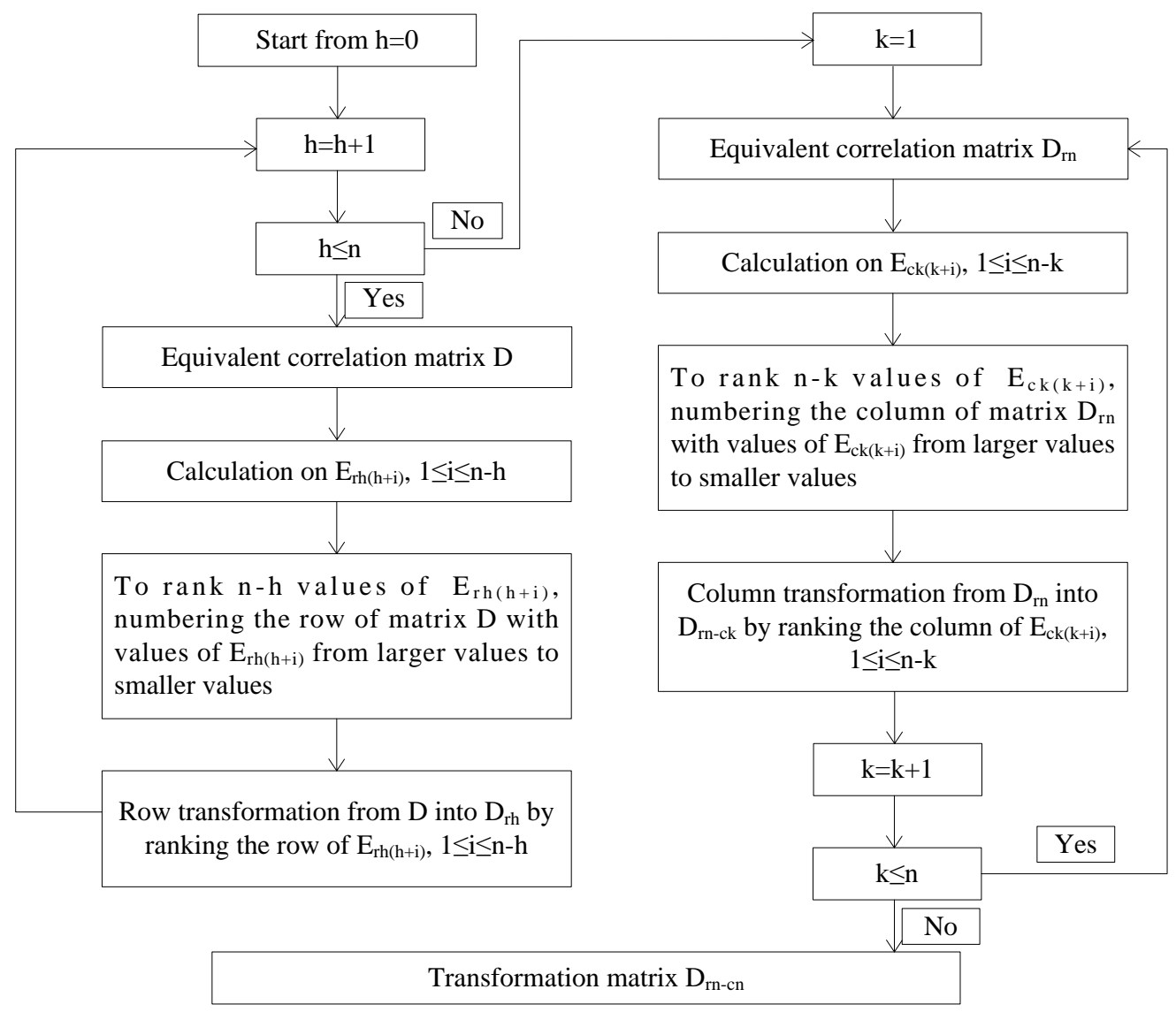

Figure 1 Flowchart of the BEA 
The processes of matrix transformation for equivalent correlation matrix $D$ using BEA are divided into row transformation and column transformation. The principles for row transformation and column transformation are the same, where the bond energy between rows and columns is calculated, respectively. The values of bond energy are compared and the rows or columns with higher bond energy will be transferred to the first forward rows or columns in matrix $D_{r n-c n}$.

\section{B. Grouping of potential sensor placements using a transformation matrix}

There are several submatrices in the transformation matrix $D_{r n-c n}$, which is the objective of matrix transformation using the BEA. The rank of each submatrix is different, which affects the following decision on the optimal sensor placement. The positions in each submatrix can be sorted as different types by the rank of each submatrix. The transformation matrix shown in Eq. (11) is taken as an example to illustrate the decision method on the type of positions. In Eq. (11), there are six submatrices in which all of the elements are equal to 1 . If there is only one element in the submatrix, the corresponding position is the Type I position. If there is only one element that is overlapping by two or more than two submatrices, the corresponding overlapping position is the Type II position. If there are more than one element in the submatrix and no elements of this submatrix belong to the other submatrix, the corresponding positions are Type III positions. If there are more than one element overlapping by two or more than two submatrices, the corresponding overlapping positions are Type IV positions. Any positions not in the aforementioned four types of positions are Type $\mathrm{V}$ positions. Based on such principles for position categories, the Type I position is $a_{1}$, the Type II position is $a_{9}$, the Type III positions are $a_{2}$ and $a_{3}$, the Type IV positions are $a_{5}$ and $a_{6}$, and the Type V positions are $a_{4}, a_{7}, a_{8}$ and $a_{10}$.

$$
\begin{array}{l|lllllllllll}
a_{1} & 1 & 0 & 0 & 0 & 0 & 0 & 0 & 0 & 0 & 0 \\
a_{2} & 0 & 1 & 1 & 0 & 0 & 0 & 0 & 0 & 0 & 0 \\
a_{3} & 0 & 1 & 1 & 0 & 0 & 0 & 0 & 0 & 0 & 0 \\
a_{4} & 0 & 0 & 0 & 1 & 1 & 1 & 0 & 0 & 0 & 0 \\
a_{5} & 0 & 0 & 1 & 1 & 1 & 1 & 1 & 0 & 0 & 0 \\
a_{6} & 0 & 0 & 1 & 1 & 1 & 1 & 1 & 0 & 0 & 0 \\
a_{7} & 0 & 0 & 0 & 1 & 1 & 1 & 1 & 0 & 0 & 0 \\
a_{8} & 0 & 0 & 0 & 1 & 1 & 1 & 1 & 1 & 1 & 0 \\
a_{9} & 0 & 0 & 0 & 0 & 0 & 0 & 0 & 1 & 1 & 1 \\
a_{10} & 0 & 0 & 0 & 0 & 0 & 0 & 0 & 0 & 1 & 1 \\
\hdashline
\end{array}
$$

\section{THE PRINCIPLE OF OPTIMAL SENSOR PLACEMENT}

\section{A. Processes of choosing optimal sensor placements}

The potential sensor placements are divided into several clustering submatrices using the BEA, the positions in which are correlated with each other more closely than the positions in other submatrices and the correlation values are all larger than the threshold value $\eta$. That is, the structural responses of any position can reflect those for the other positions in the same clustering submatrix. Thus, one position can be selected as the optimal sensor placement for each clustering submatrix to reflect the structural responses of the other placements in the same clustering submatrix. The process of choosing optimal sensor placements is as follows:

(1) If the number of Type I positions is $m_{1}$, then all of the $m_{1}$ positions are chosen as the optimal sensor placements; 
(2) If the number of Type II positions is $m_{2}$, then all of the $m_{2}$ positions are chosen as the optimal sensor placements;

(3) If the number of Type III and IV positions are $m_{3}$ and $m_{4}$, respectively, then the number of potential sensor placements within Type III and IV positions are $m_{3}^{\prime}$ and $m_{4}^{\prime}$, respectively. The total number of these four types of position is $m^{\prime}=m_{1}+m_{2}+m_{3}+m_{4}$, the number of possible combinations of optimal sensor placements depends on the number of potential sensor placements within Type III and Type IV, i.e., $m^{\prime \prime}=m_{3}^{\prime} \times m_{4}^{\prime}$. The optimal sensor placements can be determined by the minimum value of average correlation degree of all of the potential combinations; the formula for the average correlation degree of each combination is

$$
\bar{\gamma}=\frac{1}{m^{\prime 2}} \sum_{i=1}^{m^{\prime}} \sum_{j=1}^{m^{\prime}} \gamma_{i j}
$$

(4) Type V positions are always correlated to Type II or Type IV positions, whereas Type V positions are not considered in the optimal sensor positions.

(5) The optimal sensor positions determined in this step should be checked as to whether there are any optimal sensor positions in the same submatrix or if the positions in the submatrix are all correlated with the other optimal sensor positions. Such optimal sensor positions should be deleted from the selected optimal sensor positions.

\section{B. Redundancy evaluation using correlation information entropy}

The correlation information entropy $[17,18]$ is used to evaluate the redundancy between potential sensor positions. The correlation information entropy should be the smallest value for the optimal sensor placements compared to the other combinations of sensor placements. Based on the correlation matrix $R=\left\{\gamma_{i j}\right\}, 1 \leq i \leq n, 1 \leq j \leq n$, the joint information entropy $H_{R}$ can be calculated as

$$
H_{R}=-\sum_{i=1}^{n} \frac{\lambda_{i}^{R}}{n} \log _{n} \frac{\lambda_{i}^{R}}{n}
$$

where $\lambda_{i}^{R}$ is the eigenvalue of correlation matrix $R$, and the correlation information entropy $I_{R}$ is

$$
I_{R}=1-H_{R}=1+\sum_{i=1}^{n} \frac{\lambda_{i}^{R}}{n} \log _{n} \frac{\lambda_{i}^{R}}{n}
$$

The value of $I_{R}$ is between 0 and 1. $I_{R}=0$ indicates the weakest correlation between $n$ positions, i.e., the structural responses of the $n$ measuring points are independent with the lowest redundancy; $I_{R}=1$ indicates the strongest correlation between $n$ positions, and the structural responses of the $n$ measuring points are highly overlapped with the highest redundancy. Based on these characteristics of correlation information entropy, it is used to evaluate the redundancy of optimal sensor placements calculated in the paper. Higher (lower) correlation information entropy indicates greater (less) redundancy of the optimal sensor placements. The optimal sensor placements should take the smallest value of correlation information entropy when the number of the optimal sensor placements is the same.

\section{APPLICATION TO LARGE-SPAN SPATIAL STRUCTURE}

\section{A. Finite element model}

Dome-like structures can be widely found in a large variety of civil infrastructure. Without loss of generality, a Schwedler single-layer spherical lattice steel dome-like structure is considered to verify the proposed method. The finite element model of such a structure is created using the commercial finite element code ANSYS ${ }^{\circledR}$, beam4 is used to simulate the elements of the structure. The span is $50 \mathrm{~m}$, the 
sagittal height is $7.5 \mathrm{~m}$, and the rise-span ratio is $3 / 20$ with a uniform mass of $200 \mathrm{~kg} / \mathrm{m}^{2}$. The material of the elements includes a Q235 steel tube with $\phi 133 \times 4$ for the radius direction element and $\phi 127 \times 3$ for the elements in the other directions. The elastic modulus of the structure is $E=2.06 \times 10^{11} \mathrm{~N} / \mathrm{m}^{2}$, and the Poisson ratio is $\mu=0.3$. The damping ratio is $\eta=0.02$ in Rayleigh damping [19]. All of the supports are hinge fixed. The finite element model is shown in figure 2 [20].

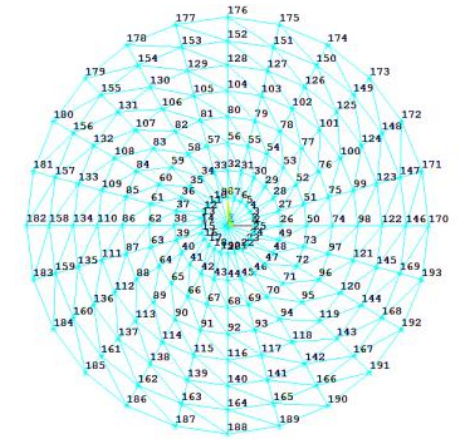

a) Vertical view of the model

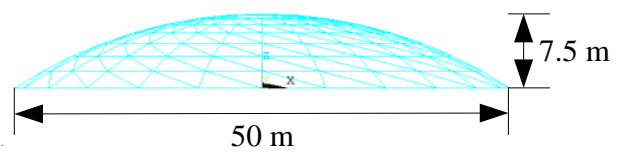

b) Lateral view of the model

Figure 2 Finite element model of the Schwedler dome-like structure

Because large-span single-layer reticulated dome-like structures are sensitive to wind load, the input load case is a wind load with an average wind speed of $5 \mathrm{~m} / \mathrm{s}$ along the horizontal $\mathrm{x}$ axis and a random fluctuating wind load with a mean value of $0 \mathrm{~m} / \mathrm{s}$ on the horizontal y axis and the vertical $\mathrm{z}$ axis. It is assumed that the optimal sensor placements should consider the monitoring work of vertical displacement, which is very important for the deformation control of the structure in both the construction phase and service phase. The monitoring work of vertical displacement can be implemented by the prisms and total station, or the laser displacement meter and so on, while the correlation analysis focuses on the vertical displacements of this dome-like structure subjected to the wind load.

\section{B. The determination of sensor number and location}

To discuss the optimal sensor placement method proposed in this paper, two scenarios of potential sensor placements are shown to process the following discussion. One scenario of potential sensor placement is shown in figure 3 . There are 24 potential sensor placements marked with solid circle dots. The other scenario of potential sensor placement is shown in figure 4 . There are 48 potential sensor placements marked with solid circle dots. The potential sensor placements in these two scenarios are simplified from the whole structure in the symmetrical position, the reason is that the potential sensor placements considered in the figures 3 and 4 are aimed to decrease the work of illustration on the proposed method, express the calculation processes and matrix exactly and completely, and discuss the determination of sensor number and location with different parameter selections.

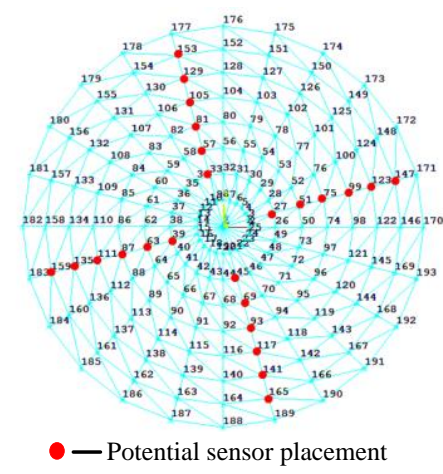

Figure 324 potential sensor placements

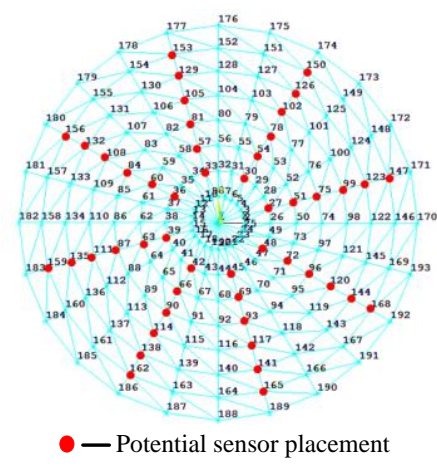

Figure 448 potential sensor placements 


\section{B1. 24 potential sensor placements}

The 24 potential sensor placements are renumbered from 1 to 24 , and the corresponding relation of the unified number and the original number for marking the potential sensor placements is shown in table 1 .

Table 1 The unified number and original number for marking the potential sensor placements

\begin{tabular}{cccccccc}
\hline $\begin{array}{c}\text { Unified } \\
\text { number }\end{array}$ & $\begin{array}{c}\text { Original } \\
\text { number }\end{array}$ & $\begin{array}{c}\text { Unified } \\
\text { number }\end{array}$ & $\begin{array}{c}\text { Original } \\
\text { number }\end{array}$ & $\begin{array}{c}\text { Unified } \\
\text { number }\end{array}$ & $\begin{array}{c}\text { Original } \\
\text { number }\end{array}$ & $\begin{array}{c}\text { Unified } \\
\text { number }\end{array}$ & $\begin{array}{c}\text { Original } \\
\text { number }\end{array}$ \\
\hline 1 & 27 & 7 & 63 & 13 & 99 & 19 & 135 \\
2 & 33 & 8 & 69 & 14 & 105 & 20 & 141 \\
3 & 39 & 9 & 75 & 15 & 111 & 21 & 147 \\
4 & 45 & 10 & 81 & 16 & 117 & 22 & 153 \\
5 & 51 & 11 & 87 & 17 & 123 & 23 & 159 \\
6 & 57 & 12 & 93 & 18 & 129 & 24 & 165 \\
\hline
\end{tabular}

(1) $\eta=0.5$

The equivalent correlation matrix $D_{0.5}$ is transformed into $D_{0.5}^{\prime}$ using the BEA. There are 10 submatrices in matrix $D_{0.5}^{\prime}$, namely, $\{23,22\},\{22,24\},\{24,21\},\{12,8\},\{8,4\},\{16,20,19,18,17,15$, $13,11,9,7,5,3,1\},\{20,19,18,17,15,13,11,9,7,5,3,1,14\},\{14,10\},\{10,6\}$, and $\{6,2\}$, which are all shown in Eq. (15). According to the sensor placement principle (1) and (2), position 22 is the overlap of $\{23,22\}$ and $\{22,24\}$, position 24 is the overlap of $\{22,24\}$ and $\{24,21\}$, position 8 is the overlap of $\{12,8\}$ and $\{8,4\}$, position 14 is the overlap of $\{20,19,18,17,15,13,11,9,7,5,3,1,14\}$ and $\{10,14\}$, position 10 is the overlap of $\{14,10\}$ and $\{6,10\}$, and position 6 is the overlap of $\{10,6\}$ and $\{6,2\}$. Positions 22, 24, 8, 14, 10 and 6 are all Type II positions, which are directly determined to be the optimal sensor placement first. In addition, positions $20,19,18,17,15,13,11,9,7,5,3$, and 1 are Type IV positions. There are 12 combinations for selecting the optimal sensor placement according to sensor placement principle (3). The average correlation degree of each combination is shown in table 2. It can be seen from table 2 that the average correlation degree of different positions shows only slight variation in value, and the minimum average value is found for the combination with position 20 . Positions 22, 24, 8, 14, 10, 6 and 20 are the selected sensor placements. With the last step of the principle of optimal sensor placement, positions 14 and 10 are from the same submatrix and should thus be deleted from the selected sensor placements. Therefore, the final optimal sensor placements are positions 22, 24 , 8, 6 and 20. According to the corresponding relation in table 1, the optimal sensor placements are 153, $165,69,57$ and 141. Their distribution in structure is shown in figure 5.

Table 2 Average values of correlation degree

\begin{tabular}{cccccc}
\hline $\begin{array}{c}\text { combination } \\
\text { with position }\end{array}$ & $\begin{array}{c}\text { average value } \\
\text { of correlation } \\
\text { degree }\end{array}$ & $\begin{array}{c}\text { combination } \\
\text { with position }\end{array}$ & $\begin{array}{c}\text { average value } \\
\text { of correlation } \\
\text { degree }\end{array}$ & $\begin{array}{c}\text { combination } \\
\text { with position }\end{array}$ & $\begin{array}{c}\text { average value } \\
\text { of correlation } \\
\text { degree }\end{array}$ \\
\hline 1 & 0.253 & 9 & 0.260 & 17 & 0.257 \\
3 & 0.265 & 11 & 0.260 & 18 & 0.263 \\
5 & 0.256 & 13 & 0.261 & 19 & 0.250 \\
7 & 0.263 & 15 & 0.258 & 20 & 0.247 \\
\hline
\end{tabular}




$$
\begin{aligned}
& 23\left[\begin{array}{llllllllllllllllllllllll}
1 & 1 & 0 & 0 & 0 & 0 & 0 & 0 & 0 & 0 & 0 & 0 & 0 & 0 & 0 & 0 & 0 & 0 & 0 & 0 & 0 & 0 & 0 & 0
\end{array}\right.
\end{aligned}
$$

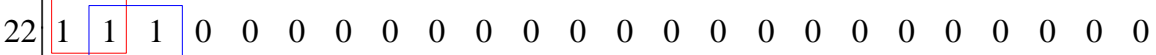

$$
\begin{aligned}
& 24 \begin{array}{lllllllllllllllllllllllll} 
& 0 & 1 & 1 & 1 & 0 & 0 & 0 & 0 & 0 & 0 & 0 & 0 & 0 & 0 & 0 & 0 & 0 & 0 & 0 & 0 & 0 & 0 & 0 & 0
\end{array} \\
& 21 \mid 0 \begin{array}{llllllllllllllllllllllll} 
& 0 & 1 & 1 & 0 & 0 & 0 & 0 & 0 & 0 & 0 & 0 & 0 & 0 & 0 & 0 & 0 & 0 & 0 & 0 & 0 & 0 & 0 & 0
\end{array}
\end{aligned}
$$

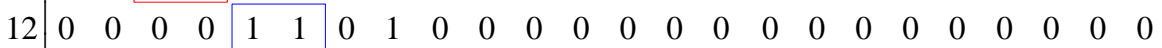

$$
\begin{aligned}
& \begin{array}{llllllllllllllllllllllllll}
8 & 0 & 0 & 0 & 0 & 1 & 1 & 1 & 0 & 0 & 0 & 0 & 0 & 0 & 0 & 0 & 0 & 0 & 0 & 0 & 0 & 0 & 0 & 0 & 0
\end{array} \\
& 4 \quad 0 \begin{array}{lllllllllllllllllllllllll} 
& 0 & 0 & 0 & 0 & 1 & 1 & 0 & 0 & 0 & 0 & 0 & 0 & 0 & 0 & 0 & 0 & 0 & 0 & 0 & 0 & 0 & 0 & 0
\end{array} \\
& 16 \mid \begin{array}{llllllllllllllllllll|llll}
0 & 0 & 0 & 0 & 1 & 0 & 0 & 1 & 1 & 1 & 1 & 1 & 1 & 1 & 1 & 1 & 1 & 1 & 1 & 1 & 0 & 0 & 0 & 0
\end{array} \\
& 20 \mid \begin{array}{llllllllllllllllllllllllll} 
& 0 & 0 & 0 & 0 & 0 & 0 & 0 & 1 & 1 & 1 & 1 & 1 & 1 & 1 & 1 & 1 & 1 & 1 & 1 & 1 & 1 & 0 & 0 & 0
\end{array} \\
& 19 \mid \begin{array}{lllllllllllllllllllllllll}
0 & 0 & 0 & 0 & 0 & 0 & 0 & 1 & 1 & 1 & 1 & 1 & 1 & 1 & 1 & 1 & 1 & 1 & 1 & 1 & 1 & 0 & 0 & 0
\end{array} \\
& 18 \mid \begin{array}{lllllllllllllllllllllllll} 
& 0 & 0 & 0 & 0 & 0 & 0 & 0 & 1 & 1 & 1 & 1 & 1 & 1 & 1 & 1 & 1 & 1 & 1 & 1 & 1 & 1 & 0 & 0 & 0
\end{array} \\
& \begin{array}{l|llllllllllllllllllllllll}
D_{0}^{\prime}=17 & 0 & 0 & 0 & 0 & 0 & 0 & 0 & 1 & 1 & 1 & 1 & 1 & 1 & 1 & 1 & 1 & 1 & 1 & 1 & 1 & 1 & 0 & 0 & 0
\end{array} \\
& D_{0.5}^{\prime}={ }_{15} \mid \begin{array}{llllllllllllllllllllllllll} 
& 0 & 0 & 0 & 0 & 0 & 0 & 0 & 1 & 1 & 1 & 1 & 1 & 1 & 1 & 1 & 1 & 1 & 1 & 1 & 1 & 1 & 0 & 0 & 0
\end{array} \\
& 13 \mid \begin{array}{lllllllllllllllllllllllll}
0 & 0 & 0 & 0 & 0 & 0 & 0 & 1 & 1 & 1 & 1 & 1 & 1 & 1 & 1 & 1 & 1 & 1 & 1 & 1 & 1 & 0 & 0 & 0
\end{array} \\
& 11 \mid \begin{array}{lllllllllllllllllllllllll}
0 & 0 & 0 & 0 & 0 & 0 & 0 & 1 & 1 & 1 & 1 & 1 & 1 & 1 & 1 & 1 & 1 & 1 & 1 & 1 & 1 & 0 & 0 & 0
\end{array} \\
& 9 \begin{array}{llllllllllllllllllllllllll}
0 & 0 & 0 & 0 & 0 & 0 & 0 & 1 & 1 & 1 & 1 & 1 & 1 & 1 & 1 & 1 & 1 & 1 & 1 & 1 & 1 & 0 & 0 & 0
\end{array} \\
& 7 \begin{array}{lllllllllllllllllllllllll}
7 & 0 & 0 & 0 & 0 & 0 & 0 & 1 & 1 & 1 & 1 & 1 & 1 & 1 & 1 & 1 & 1 & 1 & 1 & 1 & 1 & 0 & 0 & 0
\end{array} \\
& 5 \begin{array}{lllllllllllllllllllllllll}
5 & 0 & 0 & 0 & 0 & 0 & 0 & 0 & 1 & 1 & 1 & 1 & 1 & 1 & 1 & 1 & 1 & 1 & 1 & 1 & 1 & 1 & 0 & 0 & 0
\end{array}
\end{aligned}
$$

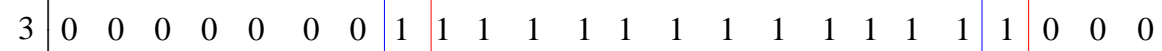

$$
\begin{aligned}
& 1 \quad \begin{array}{lllllllllllllllllllllllll} 
& 0 & 0 & 0 & 0 & 0 & 0 & 1 & 1 & 1 & 1 & 1 & 1 & 1 & 1 & 1 & 1 & 1 & 1 & 1 & 1 & 0 & 0 & 0
\end{array} \\
& 14 \mid \begin{array}{lllllllllllllllllllll|l|ll|l}
0 & 0 & 0 & 0 & 0 & 0 & 0 & 0 & 1 & 1 & 1 & 1 & 1 & 1 & 1 & 1 & 1 & 1 & 1 & 1 & 1 & 1 & 0 & 0
\end{array}
\end{aligned}
$$

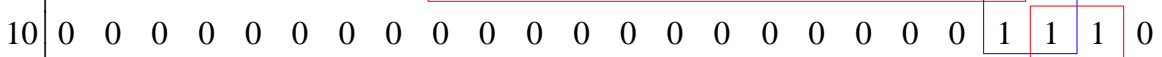

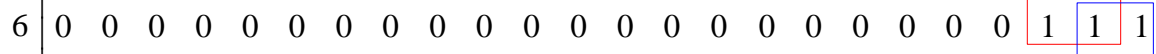

$$
\begin{aligned}
& 2\left[\begin{array}{llllllllllllllllllllllll}
0 & 0 & 0 & 0 & 0 & 0 & 0 & 0 & 0 & 0 & 0 & 0 & 0 & 0 & 0 & 0 & 0 & 0 & 0 & 0 & 0 & 0 & 1 & 1
\end{array}\right] \\
& \text { (2) } \eta=0.6
\end{aligned}
$$

The equivalent correlation matrix $D_{0.6}$ is transformed into $D_{0.6}^{\prime}$ using BEA. There are 10 submatrices in matrix $D_{0.6}^{\prime}:\{23\},\{22,24\},\{24,21\},\{12,8\},\{10,6\},\{4\},\{14,18,17,3,19,15,13,11$, $9,7,5\},\{18,17,3,19,15,13,11,9,7,5,20,1\},\{19,15,13,11,9,7,5,20,1,16\}$, and $\{2\}$. Positions 23, 4 and 2 are all Type I positions, which are directly determined to be the optimal sensor placement first. Position 24 is the overlap of $\{22,24\}$ and $\{24,21\}$ and is a Type II position; it is also directly determined as an optimal sensor placement. Positions contained in submatrices $\{8,12\}$ and $\{6,10\}$ are Type III positions. Positions $19,15,13,11,9,7$, and 5 are the overlap of three submatrices $\{14,18,17$, $3,19,15,13,11,9,7,5\},\{18,17,3,19,15,13,11,9,7,5,20,1\}$ and $\{19,15,13,11,9,7,5,20,1,16\}$, which are Type IV positions. The number of Type III positions is 4 and the number of Type IV positions is 7, respectively, whereas the discussed combinations for the optimal sensor placement are equal to $4 * 7=28$. The average values of correlation degree of each combination are shown in table 3 . It can be seen from table 3 that the average correlation degree with different positions show slight variations in value, and the minimum average value is found for the combination with position 12,10 and 19. Positions 23, 4, 2, 24, 12, 10 and 19 are the selected sensor placements. With the last step of the principle of optimal senor placement, there are no selected positions from the same submatrix, i.e., no additional position should be deleted. Therefore, the final optimal sensor placements are positions 2, 4, 10, 12, 19, 23 , and 24. According to the corresponding relation in table 1, the optimal sensor placements are 33, 45, $81,93,135,159$ and 165 ; their distribution in structure is shown in figure 6. 


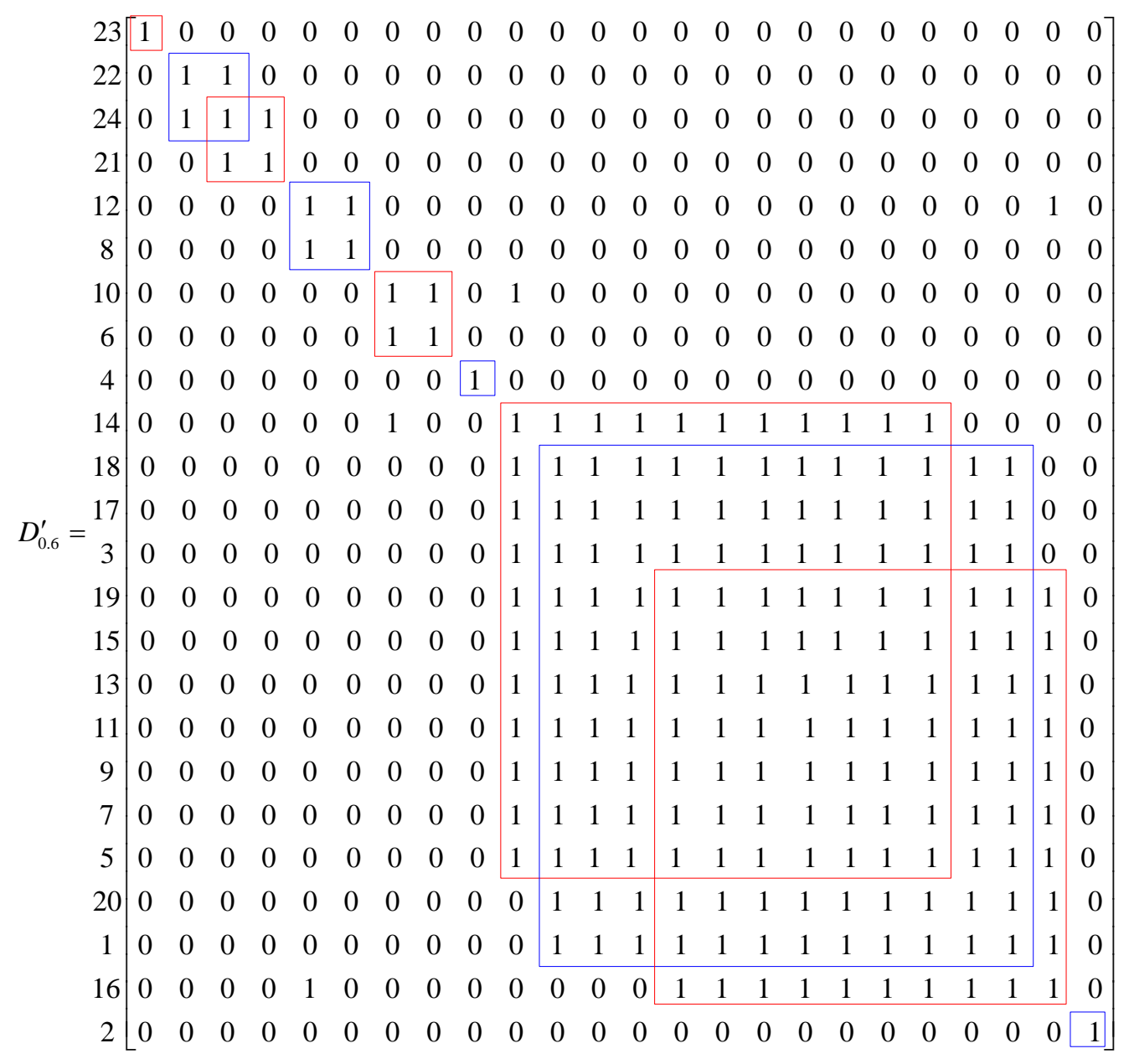

Table 3 Mean correlation degree value of each combination

\begin{tabular}{cccccccc}
\hline $\begin{array}{c}\text { combinati } \\
\text { on with } \\
\text { positions }\end{array}$ & $\begin{array}{c}\text { average } \\
\text { value of } \\
\text { correlation } \\
\text { degree }\end{array}$ & $\begin{array}{c}\text { combinati } \\
\text { on with } \\
\text { positions }\end{array}$ & $\begin{array}{c}\text { average } \\
\text { value of } \\
\text { correlation } \\
\text { degree }\end{array}$ & $\begin{array}{c}\text { combinati } \\
\text { on with } \\
\text { positions }\end{array}$ & $\begin{array}{c}\text { average } \\
\text { value of } \\
\text { correlation } \\
\text { degree }\end{array}$ & $\begin{array}{c}\text { combinati } \\
\text { on with } \\
\text { positions }\end{array}$ & $\begin{array}{c}\text { average } \\
\text { value of } \\
\text { correlation } \\
\text { degree }\end{array}$ \\
\hline $8,6,5$ & 0.198 & $12,6,5$ & 0.174 & $8,10,5$ & 0.154 & $12,10,5$ & 0.137 \\
$8,6,7$ & 0.207 & $12,6,7$ & 0.175 & $8,10,7$ & 0.168 & $12,10,7$ & 0.143 \\
$8,6,9$ & 0.203 & $12,6,9$ & 0.174 & $8,10,9$ & 0.161 & $12,10,9$ & 0.141 \\
$8,6,11$ & 0.201 & $12,6,11$ & 0.172 & $8,10,11$ & 0.161 & $12,10,11$ & 0.140 \\
$8,6,13$ & 0.203 & $12,6,13$ & 0.174 & $8,10,13$ & 0.163 & $12,10,13$ & 0.141 \\
$8,6,15$ & 0.201 & $12,6,15$ & 0.173 & $8,10,15$ & 0.160 & $12,10,15$ & 0.140 \\
$8,6,19$ & 0.197 & $12,6,19$ & 0.167 & $8,10,19$ & 0.158 & $12,10,19$ & 0.135 \\
\hline
\end{tabular}




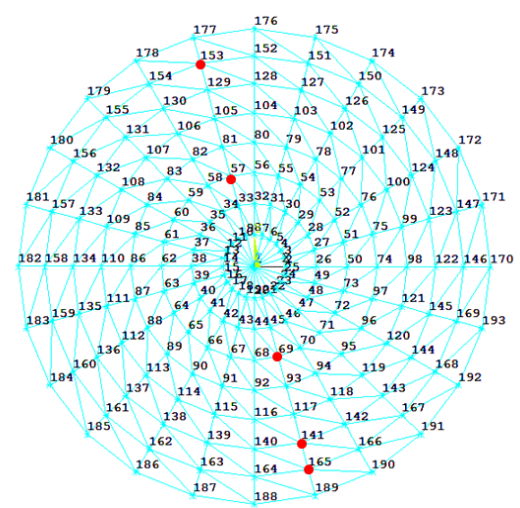

- Optimal sensor placement

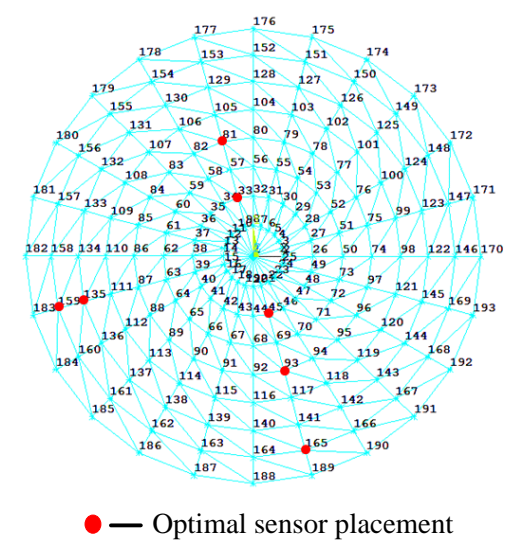

Figure 6 Optimal sensor placements with $\eta=0.6$

Figure 5 Optimal sensor placements with $\eta=0.5$

(3) When the threshold values are $\eta=0.7$ and $\eta=0.8$, the optimal sensor placements are shown as in figure 7 and figure 8 , respectively.

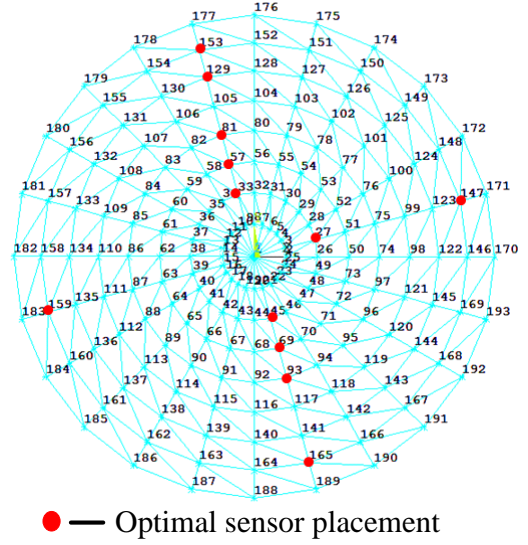

Figure 7 Optimal sensor placements with $\eta=0.7$

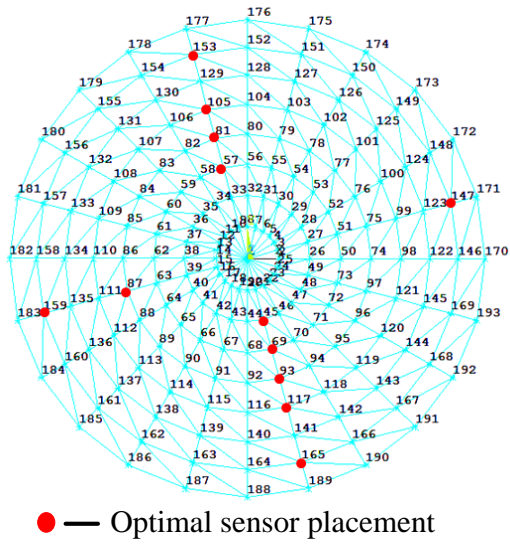

Figure 8 Optimal sensor placements with $\eta=0.8$

When the correlation degree threshold is $\eta=0.7$, the number of optimal sensor placements is 12 , and when the correlation degree threshold is $\eta=0.8$, the number of optimal sensor placements is 13 . It can be demonstrated that there are a greater number of optimal sensor placements with larger correlation degrees threshold.

\section{B2. 48 potential sensor placements}

For 48 potential sensor placements, the discussed scenarios for optimal sensor placements are $\eta=0.5, \eta=0.6, \eta=0.7$, and $\eta=0.8$. The corresponding layouts of optimal sensor placements are shown in figures 9-12. The number of optimal sensor placements for these four scenarios are 8, 10, 12 and 21 , respectively. 


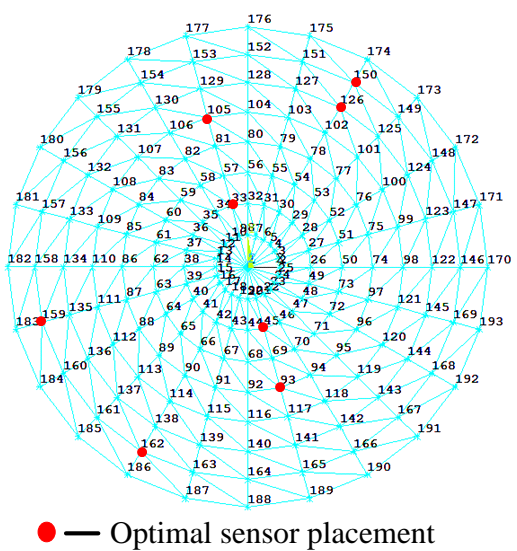

Figure 9 Optimal sensor placements with $\eta=0.5$

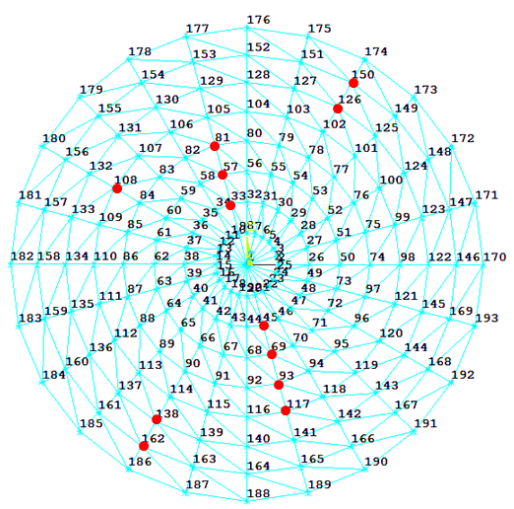

—- Optimal sensor placement

Figure 11 Optimal sensor placements with $\eta=0.7$

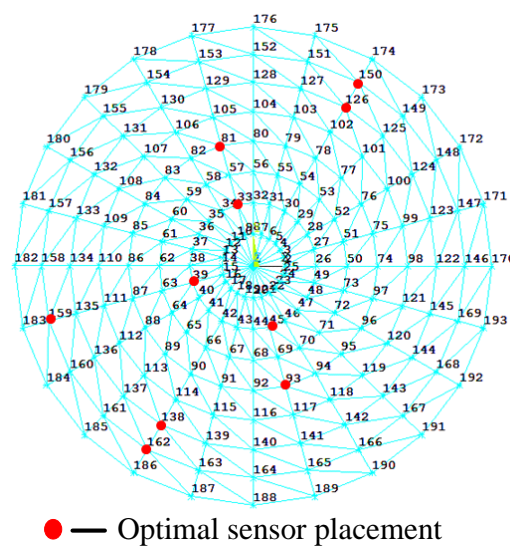

Figure 10 Optimal sensor placements with $\eta=0.6$

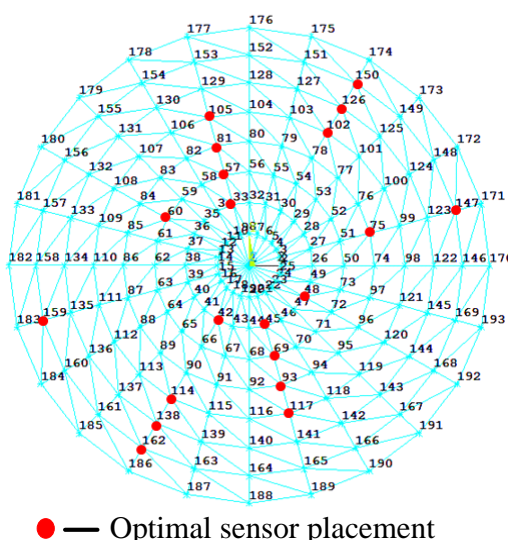

Figure 12 Optimal sensor placements with $\eta=0.8$

\section{Verification of the optimized results}

The correlation information entropy of each optimal sensor placement is calculated to evaluate the effectiveness of the proposed optimal method using the BEA. In theory, the better selection of optimal sensor placement should coincide with smaller entropy values. As an example, the number of potential sensor placements is 24 , and the thresholds are 0.5 and 0.6 , respectively. The entropy values of these two discussed scenarios are shown in table 4 and table 5. The entropy value of the combination with position 20 is the smallest, which is coincident with the selection of the aforementioned optimal sensor placements. Meanwhile, the entropy value of the combination with position 12, 10, and 19 is also the smallest, which is also coincident with the selection of the aforementioned optimal sensor placements. The proposed method of optimal sensor placements using the BEA is demonstrated by the calculation of entropy values. The analysis result indicates that the optimal placement of sensors obtained from the minimum mean correlation method all have the lowest redundancy, which satisfies the goal of maintaining the independence between sensors as much as possible. 
Table 4 Entropy value of $\eta=0.5$ for combinations

\begin{tabular}{cccccc}
\hline $\begin{array}{c}\text { Combination } \\
\text { with position }\end{array}$ & Entropy value & $\begin{array}{r}\text { Combination } \\
\text { with position }\end{array}$ & Entropy value & $\begin{array}{r}\text { Combination } \\
\text { with position }\end{array}$ & Entropy value \\
\hline 1 & 0.1048 & 9 & 0.1069 & 17 & 0.1089 \\
3 & 0.1109 & 11 & 0.1080 & 18 & 0.1066 \\
5 & 0.1080 & 13 & 0.1111 & 19 & 0.1038 \\
7 & 0.1133 & 15 & 0.1060 & 20 & 0.1028 \\
\hline
\end{tabular}

Table 5 Entropy value of $\eta=0.6$ for combinations

\begin{tabular}{cccccccc}
\hline $\begin{array}{c}\text { combination } \\
\text { with } \\
\text { positions }\end{array}$ & $\begin{array}{c}\text { Entropy } \\
\text { value }\end{array}$ & $\begin{array}{c}\text { combination } \\
\text { with } \\
\text { positions }\end{array}$ & $\begin{array}{c}\text { Entropy } \\
\text { value }\end{array}$ & $\begin{array}{c}\text { combination } \\
\text { with } \\
\text { positions }\end{array}$ & $\begin{array}{c}\text { Entropy } \\
\text { value }\end{array}$ & $\begin{array}{c}\text { combination } \\
\text { with } \\
\text { positions }\end{array}$ & $\begin{array}{c}\text { Entropy } \\
\text { value }\end{array}$ \\
\hline $8,6,5$ & 0.1087 & $12,6,5$ & 0.0804 & $8,10,5$ & 0.0826 & $12,10,5$ & 0.0631 \\
$8,6,7$ & 0.1115 & $12,6,7$ & 0.0857 & $8,10,7$ & 0.0820 & $12,10,7$ & 0.0648 \\
$8,6,9$ & 0.1082 & $12,6,9$ & 0.0812 & $8,10,9$ & 0.0824 & $12,10,9$ & 0.0636 \\
$8,6,11$ & 0.1078 & $12,6,11$ & 0.0827 & $8,10,11$ & 0.0803 & $12,10,11$ & 0.0635 \\
$8,6,13$ & 0.1094 & $12,6,13$ & 0.0835 & $8,10,13$ & 0.0804 & $12,10,13$ & 0.0628 \\
$8,6,15$ & 0.1076 & $12,6,15$ & 0.0800 & $8,10,15$ & 0.0821 & $12,10,15$ & 0.0628 \\
$8,6,19$ & 0.1051 & $12,6,19$ & 0.0765 & $8,10,19$ & 0.0798 & $12,10,19$ & 0.0593 \\
\hline
\end{tabular}

\section{Analysis of results}

The summation of optimal sensor placements with different values of correlation thresholds and different numbers of potential sensor placements are shown in table 6 and table 7 .

Table 6 Sensor quantity of different correlation degree thresholds $(n=24)$

\begin{tabular}{cccc}
\hline$\eta$ & Sensor quantity & $\eta$ & Sensor quantity \\
\hline 0.5 & 5 & 0.7 & 12 \\
0.6 & 7 & 0.8 & 13 \\
\hline
\end{tabular}

Table 7 Sensor quantity of different correlation degree thresholds $(n=48)$

\begin{tabular}{cccc}
\hline$\eta$ & Sensor quantity & $\eta$ & Sensor quantity \\
\hline 0.5 & 8 & 0.7 & 12 \\
0.6 & 10 & 0.8 & 20 \\
\hline
\end{tabular}

It can be seen from tables 4 and 5 that with higher correlation degree thresholds, there are a greater number of optimal sensor placements for each scenario of potential sensor placements. Meanwhile, when the values of the correlation degree threshold are the same for two discussed scenarios, the scenario with the greater number of potential sensor placements has more optimal sensor placements. That is, for real world application, the quantity and positions of potential sensor placements should be determined reasonably, and the correlation degree threshold should be chosen by considering the cost and the complexity of the real world structure. 


\section{CONCLUSions}

Considering the inherent correlation relationship among the structural responses, especially in terms of strain or displacement, the correlation analysis for optimal sensor placement using the BEA is proposed. The correlation matrix of potential sensor placement is built and the correlation submatrices are obtained using the BEA. The potential sensor placements in the same submatrix are the placements with the highest correlation evaluated by the correlation degree threshold. The optimal sensor placements determined by this proposed method are correlated with all of the potential sensor placements within the selected correlation degree threshold. Therefore, the structural response variations of these optimal sensor placements can reflect those of all of the potential sensor placements. A Schwedler single-layer dome-like steel structure is used to verify the proposed method by comparing the entropy value of each discussed scenario. The number of optimal sensor placements depends on the initial number of potential sensor placements and the correlation degree threshold. The number of optimal sensor placements should be determined by considering the cost and complexity of the structure, for which the proposed method can effectively determine optimal sensor placements and reduce the redundancy of the monitoring structural responses.

\section{ACKNOWLEDGMENTS}

This research is supported by the National Science Foundation of China (Grant No. 51308162), the Shenzhen Knowledge Innovation Program - Fundamental Research (JCYJ20140417172417117), the Natural Scientific Research Innovation Foundation at the Harbin Institute of Technology (HIT.NSRIF.2015085), and the Supporting Project for Junior Faculty of the Harbin Institute of Technology Shenzhen Graduate School.

\section{References}

[1] H. X. He, W. M Yan, A. L. Zhang, Optimization of number and placement of sensors for structural health monitoring, J. Vib. Shock 27(9) (2008) 131-134.

[2] W. Liu, W. C. Gao, Y. Sun, M. J. Xu, Optimal sensor placement for spatial lattice structure based on genetic algorithms, J. Sound Vib. 317(1-2) (2008) 175-189.

[3] J. Teng, Y. H. Zhu, Optimal sensor placement for modal parameters test of large span spatial steel structural, Eng. Mech. 28(3) (2011) 150-156.

[4] F. E Udwadia, Methodology for optimal sensor locations for parameter identification in dynamic systems, J. Eng. Mech. 120(2) (1994) 368-390.

[5] D. C. Kammer, Sensor placement for on-orbit modal identification and correlation of large space structures, J. Guid. Control Dynam. 14(2) (1991) 251-259.

[6] T. Breitfeld, A Method for identification of a set of optimal measurement points for experimental modal analysis, Modal Anal. 11(1-2) (1996) 1-9.

[7] H Baruh, K Choe, Sensor-failure detection method for flexible structure, J. Guid. Control Dynam. 10(5) (1987) 474-482.

[8] F. Cui, W. C. Yuan, Application of optimal sensor placement algorithms for health monitoring of bridges, J. Tongji Univ. 27(2) (1999) 165-169.

[9] R. J. Guyan, Reduction of stiffness and mass matrices, AIAA J. 3(2) (1965) 380.

[10] W. Lu, J. Teng, Optimal placement of sensors based on data fusion, J. Vib. Shock, 28(9) (2009) $52-55$.

[11] C. D. Zhang, Y. L. Xu. Optimal multi-type sensor placement for response and excitation reconstruction, J. Sound Vib. 360 (2016) 112-128.

[12] Y. H. Teo, W. K. Chiu, F. K. Chang, N. Rajic. Optimal placement of sensors for sub-surface fatigue crack monitoring, Theor. Appl. Fract. Mec. 52 (2009) 40-49. 
[13] G. D’Antona, N. Seifnaraghi. Analysis of the sensor placement for optimal temperature distribution reconstruction, Measurement 56 (2014) 58-69.

[14] J. M. Beal, A. Shukla, O. A. Brezhneva, M. A. Abramson. Optimal sensor placement for enhancing sensitivity to change in stiffness for structural health monitoring, Optim. Eng. 9 (2008) 119-142.

[15] W. Chen, W. G. Zhao, H. P. Zhu, J. F. Chen. Optimal sensor placement for structural response estimation, J. Cent. South Univ. 21 (2014) 3993-4001.

[16] S. Dowlatshahi, A modeling approach to logistics in concurrent engineering, Eur. J. Oper. Res. 115(1) (1999) 59-76.

[17] Q. Wang, Y. Shen, Y. Zhang, J. Q. Zhang, Fast quantitative correlation analysis and information deviation analysis for evaluating the performances of image fusion techniques, IEEE T. Instrum. Meas. 53(5) (2004) 1441-1447.

[18] Q. Wang, Y. Shen, J. Q. Zhang, A nonlinear correlation measure for multivariable data set, Physica D 200(3-4) (2005) 287-295.

[19] E. L Wilson, Three Dimensional Static and Dynamic Analysis of Structures, Berkeley, CA: Computers and Structures, 2002

[20] W. Lu, J. Teng, Y. L. Xu, Z. Q. Su, Identification of damage in dome-like structures using hybrid sensor measurements and artificial neural networks, Smart Mater. Struct. 22(10) (2013) 5-14. 